\title{
On the Chaplygin sphere in a magnetic field
}

\author{
A.V.Borisov ${ }^{(1)}$, A.V. Tsiganov ${ }^{(2)}$ \\ (1) Udmurt State University; \\ (2) Steklov Mathematical Institute, Russian Academy of Sciences; \\ borisov@rcd.ru, andrey.tsiganov@gmail.com
}

\begin{abstract}
We consider the possibility of using Dirac's ideas of the deformation of Poisson brackets in nonholonomic mechanics. As an example, we analyze the composition of external forces that do no work and reaction forces of nonintegrable constraints in the model of a nonholonomic Chaplygin sphere on a plane. We prove that, when a solenoidal field is applied, the general mechanical energy, the invariant measure and the conformally Hamiltonian representation of the equations of motion are preserved. In addition, we consider the case of motion of the nonholonomic Chaplygin sphere in a constant magnetic field taking dielectric and ferromagnetic (superconducting) properties of the sphere into account. As a by-product we also obtain two new integrable cases of the Hamiltonian rigid body dynamics in a constant magnetic field taking the magnetization by rotation effect into account.
\end{abstract}

\section{Introduction}

It has been known since as far back as the 1940s that the equations of motion of a dielectric rigid body about a fixed point in a homogeneous and stationary magnetic field are represented as Euler-Poisson equations with some generalized potential. This problem received particular attention in the work of Grioli [21, 22, 3] in which it was emphasized that the work of external forces in this case is zero. For this reason it is often called the Grioli problem.

The equations of motion that appear in the Grioli problem are Hamiltonian equations, and one can also add to the corresponding Hamiltonian quadratic terms due to the Newtonian potential, the Brun field or fast vibrations of the suspension point [10. In this case, the equations have the form of Kirchhoff equations describing the motion of a singly connected body in an infinite volume of an ideal incompressible fluid which is at rest at infinity. A detailed discussion of the integrability by quadratures of the Kirchhoff equations with various potential and gyroscopic terms can be found in the book [10].

If we add gyrostatic terms to the Grioli problem, then, from the viewpoint of motion in a fluid, they can be treated as multiple connection of a rigid body, which admits circulation around contours. This analogy and the generalization of these Hamiltonian equations to the $n$-dimensional case is discussed in detail in the book 8 .

Another, not less important, problem is that of the motion of a ferromagnetic or superconducting body in a magnetic field with magnetization during rotation [1, 2, 23, 26, 27, 32, 37. In contrast to the Grioli problem, in the general case the equations of motion are not Hamiltonian and, generally speaking, possess no invariant measure and do not preserve the total mechanical energy of the body. After the change of variables, all well-known integrable particular cases [32, 27, in this problem are reduced either to the integrable Kirchhoff case [27] or to the integrable Clebsch case [38] in the Kirchhoff equations, see Appendix D in the book [10].

In this paper we consider the nonholonomic problem of the motion of an inhomogeneous dynamically balanced sphere rolling without slipping on a horizontal plane with its center of mass at the geometric center. This is the so-called Chaplygin sphere. Chaplygin found an invariant measure and first integrals for the equations of motion of the sphere in the gravitational field and reduced the problem to quadratures. By the way, this year marks the 150th birthday of this great Russian mechanical 
engineer. The generalization presented here is related to a sphere that exhibits both dielectric and ferromagnetic properties and is acted upon by potential forces. We note that in the case of fast vibrations of the plane a quadratic potential can appear and the study of such problems holds much promise for modern dynamics from the viewpoint of vibrostabilization and vibrodisplacement.

As a rule, the equations of motion of such systems cannot be represented in Hamiltonian form, but can be reduced to it after conformal transformation of coordinates and time. In addition to investigating the first integrals and invariant measures, we present a general construction which makes it possible to include magnetic terms in the conformally Hamiltonian representation of the equations of motion. Thus, we present general cases of existence of additional integrals, an invariant measure and a conformally Hamiltonian representation for equations which, due to hydrodynamical analogy, can be called nonholonomic Kirchhoff equations.

Other types of nonholonomic Kirchhoff equations with two degrees of freedom are dealt with in [18, 19. In these papers, some "knife edges" are added to the standard 2D hydrodynamical problem and the equations thus obtained describe the hydrodynamical Chaplygin sleigh. However, generally speaking, such an attachment of knife edges in an ideal fluid cannot be done from a mechanical point of view, and the problem is described not by nonholonomic, but vakonomic equations, see the review [12. In our problem, we use standard equations of rolling of a rigid body in a magnetic field, and therefore no additional nonrealistic hypotheses are required.

The paper is organized as follows. The rest of Section 1, devoted to Dirac's development of Hamiltonian mechanics, includes a discussion of the energy paradigm and deformation of the Poisson brackets. In Section 2 we construct a conformally Hamiltonian vector field describing the motion of the Chaplygin sphere in an arbitrary solenoidal field. In the particular case of magnetic forces we obtain a nonholonomic analog of the Grioli problem [21, 22, see also [3] and the textbooks [8, 10. Section 3 is devoted to discussion of the nonholonomic analog of the classical Grioli and Barett-London problems for the Chaplygin sphere. For instance, we consider the existence of an invariant measure and of integrals of motion for a body which consists of dielectric and ferromagnetic parts. As a by-product we also obtain two new integrable cases of Hamiltonian rigid body dynamics in a magnetic field.

\subsection{Magnetic field in a Dirac approach}

The passage from Lagrangian to Hamiltonian mechanics via a Legendre transformation is inadequate in several specific situations:

- when the Lagrangian is a linear function in the velocity;

- when there are gauge or other unphysical degrees of freedom;

- when there are constraints that one wishes to impose in phase space.

For instance, let us take a particle with charge $q$ confined to the $x y$ plane with a strong constant, homogeneous perpendicular magnetic field pointing in the $z$-direction with strength $B$ [17]. In the limit of a very large magnetic field one may drop the kinetic term to produce a simple approximate Lagrangian

$$
L=\frac{q B}{2 c}(x \dot{y}-y \dot{x})-V(x, y) .
$$

The corresponding equations of motion are

$$
\dot{y}=\frac{c}{q B} \frac{\partial V}{\partial x}, \quad \dot{x}=-\frac{c}{q B} \frac{\partial V}{\partial y},
$$

where $c$ is the speed of light in vacuum and $V(x, y)$ is an arbitrary external scalar potential. Note that this approximate Lagrangian is linear in the velocities, which is one of the conditions under which the standard Hamiltonian procedure breaks down.

Following the Hamiltonian procedure, however, the canonical momenta associated with the coordinates are now

$$
p_{x}=\frac{\partial L}{\partial \dot{x}}=-\frac{q B}{2 c} y, \quad p_{y}=\frac{\partial L}{\partial \dot{y}}=\frac{q B}{2 c} x,
$$


which are unusual in that they are not invertible to the velocities. Instead, they are constrained to be functions of the coordinates. A Legendre transformation then produces the Hamiltonian

$$
H\left(x, y, p_{x}, p_{y}\right)=\dot{x} p_{x}+\dot{y} p_{y}-L=V(x, y) .
$$

It is easy to see that this Hamiltonian has no dependence on the momenta, which means that Hamilton's equations of motion are inconsistent. For a complete discussion, see [17.

In [14, 15, 16] Dirac argues that we should generalize the Hamiltonian somewhat analogously to the method of Lagrange multipliers and deform Poisson bracket in order to treat classical systems with second-class constraints in Hamiltonian mechanics, and to thus allow them to undergo canonical quantization. It is an important part of Dirac's development of Hamiltonian mechanics to elegantly handle more general Lagrangians, see also 20] for a discussion of possible generalizations.

Let us briefly recall the main ideas of Dirac's development of Hamiltonian mechanics. In Newtonian mechanics the addition of the external force $F^{\prime}$ changes the equation of motion

$$
m \ddot{q}_{i}=F_{i} \rightarrow m \ddot{q}_{i}=F_{i}+F^{\prime}, \quad i=1, \ldots, n
$$

where $q_{i}$ are coordinates on a configurational space.

In the Lagrangian formulation, we have the function $L\left(q_{i}, \dot{q}_{i}, t\right)$, where $q_{i}$ are generalized coordinates and the equations of motion are

$$
\frac{\mathrm{d}}{\mathrm{d} t}\left(\frac{\partial L}{\partial \dot{q}_{i}}\right)-\frac{\partial L}{\partial q_{i}}=0
$$

In order to change these equations, we have to shift $L$ by generalized potential $U\left(q_{i}, \dot{q}_{i}\right)$

$$
L\left(q_{i}, \dot{q}_{i}, t\right) \rightarrow L^{\prime}\left(q_{i}, \dot{q}_{i}, t\right)=L\left(q_{i}, \dot{q}_{i}, t\right)-U\left(q_{i}, \dot{q}_{i}\right) .
$$

The Lagrangian $L\left(q_{i}, \dot{q}_{i}, t\right)$ is a function of the coordinates $q_{i}$, their time derivatives $\dot{q}_{i}$ and time.

The basic idea of Hamilton's approach is to introduce generalized momenta

$$
p_{i}=\frac{\partial L}{\partial \dot{q}_{i}}
$$

and Hamiltonian $H$ which is the Legendre transform of the Lagrangian $L$ with respect to the $\dot{q}_{i}$ variables

$$
H\left(q_{i}, p_{i}, t\right)=\sum_{i=1}^{n} p_{i} \dot{q}_{i}-L\left(q_{i}, \dot{q}_{i}, t\right) .
$$

Here $\dot{q}_{i}$ is eliminated from the right-hand side in favor of $p_{i}$. Thus, in Hamiltonian formalism equations of motion are defined by the Hamiltonian function $H$ and by the Poisson bivector $P$

$$
\frac{\mathrm{d} x_{i}}{\mathrm{~d} t}=X_{i}, \quad X=P \mathrm{~d} H,
$$

where $x=\left(q_{1}, \ldots, q_{n}, p_{1}, \ldots, p_{n}\right)$ is a point in $2 n$-dimensional phase space. Together with the Poisson bivector $P$ we use the Poisson brackets defined by

$$
\{f(x), g(x)\}=(\mathrm{d} f, P \mathrm{~d} g), \quad \mathrm{d} f=\left(\frac{\mathrm{d} f}{\mathrm{~d} x_{1}}, \frac{\mathrm{d} f}{\mathrm{~d} x_{2}}, \ldots, \frac{\mathrm{d} f}{\mathrm{~d} x_{n}}\right),
$$

where $f$ and $g$ are two functions on the phase space and $(.,$.$) means a scalar product of two 2 n$ dimensional vectors.

Addition of external force replaces the original momenta $p_{i}$ to generalized momenta

$$
p_{i} \rightarrow \tilde{p}_{i}=\frac{\partial L}{\partial \dot{q}_{i}}-\frac{\partial U}{\partial \dot{q}_{i}}=p_{i}-\frac{\partial U}{\partial \dot{q}_{i}}
$$

and changes the Hamiltonian

$$
H\left(q_{i}, p_{i}, t\right) \rightarrow H^{\prime}\left(q_{i}, p_{i}, t\right)=H\left(q_{i}, p_{i}, t\right)+U\left(q_{i}, p_{i}\right) .
$$


If the original Hamiltonian $H$ is the total mechanical energy and the work done by external forces is equal to zero, the total mechanical energy $H$ is altered and the new Hamiltonian $H^{\prime}$ has no mechanical meaning.

According to Dirac, we can preserve the energy paradigm by saying that the total mechanical energy contains all the dynamical information and mathematical Hamiltonian formalism by using deformation of the Poisson brackets. In Dirac's approach the addition of nonconservative external force changes the original equations of motion (1.1) to equations of the form

$$
\frac{\mathrm{d} x_{i}}{\mathrm{~d} t}=X_{i}^{\prime}, \quad X^{\prime}=P^{\prime} \mathrm{d} H
$$

instead of equivalent equations

$$
\frac{\mathrm{d} x_{i}}{\mathrm{~d} t}=X_{i}^{\prime}, \quad X^{\prime}=P \mathrm{~d} H^{\prime},
$$

which follow from the Legendre transformation of the generalized Lagrangian $L^{\prime}=L-U$. Having the equations of motion, however, is not the endpoint for theoretical considerations. If one wants to canonically quantize a general system or study its other deformations, then one needs the Dirac brackets.

Thus, in Hamiltonian formalism we can change equations of motion (1.1) by using

- deformation of Hamiltonian $H \rightarrow H^{\prime}$,

- deformation of the Poisson bivector $P \rightarrow P^{\prime}$,

which both are related to transformation of momenta $p_{i}$ to generalized momenta $\tilde{p}_{i}$. Deformations of the Poisson brackets associated with a magnetic (solenoidal) field are discussed in [31].

In this paper we discuss the application of the Dirac idea in nonholonomial mechanics. As an example, we consider a nonholonomic Chaplygin sphere in a magnetic (solenoidal) field. In this case we have to study the composition of a known deformation associated with reaction forces of the nonholonomic constraint [1] with a known deformation associated with a magnetic field [4, 31].

\section{Deformations of the Poisson brackets}

Let us consider a Lie-Poisson algebra $e^{*}(3)$ endowed with the linear Poisson brackets

$$
\left\{\gamma_{i}, \gamma_{j}\right\}=0 . \quad\left\{M_{i}, \gamma_{j}\right\}=\varepsilon_{i j k} \gamma_{k}, \quad\left\{M_{i}, M_{j}\right\}=\varepsilon_{i j k} M_{k}
$$

where $\varepsilon_{i j k}$ is a complete antisymmetric tensor. The corresponding Lie-Poisson bivector is equal to

$$
P=\left(\begin{array}{ll}
0 & \Gamma \\
\Gamma & \mathrm{M}
\end{array}\right)
$$

Here $\gamma=\left(\gamma_{1}, \gamma_{2}, \gamma_{3}\right)$ and $M=\left(M_{1}, M_{2}, M_{3}\right)$ are vectors in three-dimensional Euclidean space $\mathbb{R}^{3}$, which can be identified with $3 \times 3$ skew-symmetric matrices in $s o(3)$

$$
\Gamma=\left(\begin{array}{ccc}
0 & \gamma_{3} & -\gamma_{2} \\
-\gamma_{3} & 0 & \gamma_{1} \\
\gamma_{2} & -\gamma_{1} & 0
\end{array}\right), \quad \mathrm{M}=\left(\begin{array}{ccc}
0 & M_{3} & -M_{2} \\
-M_{3} & 0 & M_{1} \\
M_{2} & -M_{1} & 0
\end{array}\right)
$$

using the standard isomorphism of Lie algebras $\left(\mathbb{R}^{3}, a \times b\right)$ and $(s o(3),[A, B])$, where $a \times b$ is a vector product and $[A, B]$ is a matrix commutator.

Bivector $P$ has two Casimir functions:

$$
C_{1}=(\gamma, \gamma), \quad C_{2}=(\gamma, M), \quad P \mathrm{~d} C_{1}=0, \quad P \mathrm{~d} C_{2}=0,
$$

and, together with the Hamiltonian

$$
H=\frac{1}{2}(M, \omega)+V(\gamma)
$$


generates the Hamiltonian vector field

$$
X=P \mathrm{~d} H
$$

If we identify coordinates $M$ with the angular momentum vector of the rigid body, $\gamma$ with the unit Poisson vector in the rigid body frame and $H$ with the total mechanical energy of the rigid body, then this vector field corresponds to the standard Euler-Poisson equations describing the rotation of the rigid body around a fixed point in a potential field

$$
\dot{\gamma}=\gamma \times \omega, \quad \dot{M}=M \times \omega-\frac{\partial V}{\partial \gamma} \times \gamma .
$$

Here $\omega=A M$ is the angular velocity vector, $A=I^{-1}=\operatorname{diag}\left(a_{1}, a_{2}, a_{3}\right)$ is an inverse matrix to the tensor of inertia $I$. All the vectors are expressed in the so-called body frame, which is firmly attached to the rigid body, its origin is at the center of mass of the body, and its axes coincide with the principal inertia axes of the body $[\underline{8}, 10$.

\subsection{Magnetic Poisson brackets}

Construction of the so-called magnetic Poisson structures which are deformations of the standard LiePoisson brackets on the various Lie algebras may be found in [31]. Here we discuss only a partial deformation of the Poisson brackets (2.3) on $e^{*}(3)$ which is related to the well-known transformation of the original angular momentum

$$
\varphi: \quad M_{i} \rightarrow \tilde{M}_{i}=M_{i}+c_{i}\left(\gamma_{1}, \gamma_{2}, \gamma_{3}\right), \quad i=1,2,3,
$$

to the generalized angular momentum $\tilde{M}_{i}$, which allows us to present the generalized Hamiltonian function

$$
H^{\prime}(q, p)=T(q, p)+V(q)+U(q, p)
$$

as a sum of generalized kinetic energy and potential

$$
H^{\prime}(q, p)=T^{\prime}(q, p)+V(q)
$$

see [8] for details and references therein.

Mapping (2.7) reduces the original linear Lie-Poisson brackets (2.3) to the following Poisson brackets:

$$
\left\{\gamma_{i}, \gamma_{j}\right\}=0 . \quad\left\{M_{i}, \gamma_{j}\right\}=\varepsilon_{i j k} \gamma_{k}, \quad\left\{M_{i}, M_{j}\right\}=\varepsilon_{i j k}\left(M_{k}+b_{k}\right)
$$

where

$$
\begin{aligned}
& b_{1}=\left(\frac{\partial c_{2}}{\partial \gamma_{2}}+\frac{\partial c_{3}}{\partial \gamma_{3}}\right) \gamma_{1}-\frac{\partial\left(\gamma_{2} c_{2}+\gamma_{3} c_{3}\right)}{\gamma_{1}}-c_{1}, \\
& b_{2}=\left(\frac{\partial c_{1}}{\partial \gamma_{1}}+\frac{\partial c_{3}}{\partial \gamma_{3}}\right) \gamma_{2}-\frac{\partial\left(\gamma_{1} c_{1}+\gamma_{3} c_{3}\right)}{\partial \gamma_{2}}-c_{2}, \\
& b_{3}=\left(\frac{\partial c_{1}}{\partial \gamma_{1}}+\frac{\partial c_{2}}{\partial \gamma_{2}}\right) \gamma_{3}-\frac{\partial\left(\gamma_{1} c_{1}+\gamma_{2} c_{2}\right)}{\partial g_{3}}-c_{3}
\end{aligned}
$$

so that

$$
(\operatorname{rot} b, \gamma)=(\nabla \times b, \gamma)=0 \text {. }
$$

Here $b=\left(b_{1}, b_{2}, b_{3}\right)$ is a vector depending on coordinates $\gamma$. Associated with the Poisson brackets (2.8) is the deformation of the Poisson bivector, which has the form

$$
P_{\varphi}=\left(\begin{array}{cc}
0 & \Gamma \\
\Gamma & \mathrm{M}+\mathrm{B}
\end{array}\right), \quad \mathrm{B}=\left(\begin{array}{ccc}
0 & b_{3} & -b_{2} \\
-b_{3} & 0 & b_{1} \\
b_{2} & -b_{1} & 0
\end{array}\right)
$$

The corresponding deformations of the Casimir functions (2.5) read as

$$
C_{1}=(\gamma, \gamma), \quad C_{2}=(\gamma, M-c), \quad P_{\varphi} \mathrm{d} C_{1}=0, \quad P_{\varphi} \mathrm{d} C_{2}=0
$$


Let us describe an inverse transformation $P_{\phi} \rightarrow P$ and suppose that we have bivector $P_{\varphi}$ (2.11) with arbitrary functions $b_{1}, b_{2}$ and $b_{3}$. This bivector satisfies the Jacobi condition only if these functions satisfy condition (2.10)

$$
(\operatorname{rot} b, \gamma)=(\nabla \times b, \gamma)=0 .
$$

However, substituting functions $b_{1}, b_{2}$ and $b_{3}$ into (2.9), we obtain an inconsistent system of differential equations with respect to functions $c_{1}, c_{2}$ and $c_{3}$ even if (2.10) holds. These equations become compatible only if we suppose that $P_{\phi}$ has two Casimir functions linear in momenta $M_{i}$.

Lemma 1 Bivector $P_{\phi}$ (2.11) depending on arbitrary functions $b_{1}, b_{2}$ and $b_{3}$ of coordinates $\gamma_{1}, \gamma_{2}, \gamma_{3}$ is a Poisson bivector with two Casimir functions linear in $M_{i}$

$$
C_{1}=(\gamma, \gamma), \quad C_{2}=(\gamma, M)+e\left(\gamma_{1}, \gamma_{2}, \gamma_{3}\right)
$$

if $b_{1}\left(\gamma_{1}, \gamma_{2}, \gamma_{3}\right)$ and $e\left(\gamma_{1}, \gamma_{2}, \gamma_{3}\right)$ are arbitrary functions, whereas

$$
b_{2}=\frac{\gamma_{2}}{\gamma_{1}}\left(b_{1}(\gamma)-\frac{\partial e(\gamma)}{\left.\partial \gamma_{1}\right)}\right)+\frac{\partial e(\gamma)}{\partial \gamma_{2}}, \quad b_{3}=\frac{\gamma_{3}}{\gamma_{1}}\left(b_{1}(\gamma)-\frac{\partial e(\gamma)}{\partial \gamma_{1}}\right)+\frac{\partial e(\gamma)}{\partial \gamma_{3}},
$$

up to permutation of the indices of $b_{k}$.

Now, substituting functions $b_{1,2}$ (2.13) into Eqs. (2.9), we obtain a compatible system of differential equations of second order on $c_{1}, c_{2}$ and $c_{3}$. The generic solution of these equations may be obtained by using a computer algebra system. For brevity we do not present this solution here.

The new Poisson bivector $P_{\phi}$ (2.11) and the original mechanical energy $H$ (2.6) generate the Hamiltonian vector field

$$
X_{\varphi}=P_{\varphi} \mathrm{d} H, \quad H=\frac{1}{2}(M, \omega)+V(\gamma),
$$

associated with the Euler-Poisson equations

$$
\dot{\gamma}=\gamma \times \omega, \quad \dot{M}=(M+b) \times \omega-\frac{\partial V}{\partial \gamma} \times \gamma,
$$

describing the rotation of the rigid body about fixed points in a potential field with potential $V(\gamma)$ and in a solenoidal field $\gamma \times b$, because

$$
\operatorname{div} \gamma \times b=0
$$

according to (2.10).

At $b=B \gamma+\alpha$ the vector field $X_{\varphi}$ describes the dynamics of the charged rigid body with stationary charge distribution (dielectric) rotating about the fixed point in a constant magnetic field, for details about this Grioli problem, see the textbooks [8, 10]. In this case the symmetric matrix $B$ describes an electric charge distribution, whereas the vector $\alpha$ is a vector of gyrostatic momentum. Similar equations appear in the description of an underwater vehicle when matrix $B$ describes a buoyancy distribution [31.

Substituting into the definition of the vector field $X_{\varphi}$ various Hamiltonians $H$ associated with the well-known integrable cases of rigid body motion, one gets Euler, Lagrange, Kowalewski tops or Clebsch and Steklov - Lyapunov systems in a solenoidal field, but in the general case the addition of a magnetic field destroys integrability of the original dynamical systems [4, 10, 31, 27.

\subsection{Nonholonomic magnetic Poisson brackets}

In [5, 6, 9, 11, 34, 36] we construct linear in $M_{i}$ transformations of angular momentum

$$
\psi: \quad M_{i} \rightarrow \tilde{M}_{i}=f_{i}(\gamma) M_{i}+g_{i}(\gamma)
$$

which reduce the original Lie-Poisson bivector $P(2.4)$ to a Poisson bivector $P_{\psi}$, which allows us to decompose the non-Hamiltonian vector field by Hamiltonian vector fields. It allows us to use methods of Hamiltonian mechanics to study non-Hamiltonian systems such as the Chaplygin sphere on a plane, 
a sphere and a turntable, other generalizations of the Chaplygin sphere model, the Routh sphere model, the Veselova system and many other nonholonomic systems.

It is natural to study the decomposition of the mappings $\varphi(2.7)$ and $\psi(2.14)$ which corresponds to a simultaneous addition of the external nonconservative forces and reaction forces associated with nonintegrable constraints. In this note we consider the transformation of $M_{i}$ variables

$$
\psi:\left\{\begin{array}{l}
M_{1} \rightarrow \tilde{M}_{1}=g\left(M_{1}-\frac{\beta \gamma_{1}}{\gamma_{1}^{2}+\gamma_{2}^{2}}\right)+\frac{\alpha \gamma_{1}}{(\gamma, \gamma)}\left(1+\frac{\gamma_{3}^{2}}{\nu}\right), \\
M_{2} \rightarrow \tilde{M}_{2}=g\left(M_{2}-\frac{\beta \gamma_{2}}{\gamma_{1}^{2}+\gamma_{2}^{2}}\right)+\frac{\alpha \gamma_{2}}{(\gamma, \gamma)}\left(1+\frac{\gamma_{3}^{2}}{\nu}\right), \\
M_{3} \rightarrow \tilde{M}_{3}=g M_{3}+\frac{\alpha \gamma_{3}}{(\gamma, \gamma)}\left(1-\frac{\gamma_{1}^{2}+\gamma_{2}^{2}}{\nu}\right),
\end{array}\right.
$$

where

$$
\alpha=(\gamma, M), \quad \beta=(\gamma, L) \quad \text { and } \quad \nu=\gamma_{1}^{2}+\gamma_{2}^{2}-d(\gamma, \gamma)\left(a_{1} \gamma_{1}^{2}+a_{2} \gamma_{2}^{2}\right) .
$$

This mapping and the corresponding deformation of the Lie-Poisson bivector $P$ (2.4) depend on parameter $d$ and the diagonal matrix $A=\operatorname{diag}\left(a_{1}, a_{2}, a_{3}\right)$, which determine the function

$$
g=\sqrt{1-d(\gamma, A \gamma)} .
$$

Mapping $\psi(2.15)$ reduces the original Lie-Poisson bivector $P$ (2.4) to the following Poisson bivector:

$$
P_{\psi}=g P-\frac{d}{g}(M, A \gamma)\left(\begin{array}{cc}
0 & 0 \\
0 & \Gamma
\end{array}\right),
$$

and preserves the form of the original Casimir functions (2.5)

$$
C_{1}=(\gamma, \gamma), \quad C_{2}=(\gamma, M), \quad P_{\psi} \mathrm{d} C_{1}=0, \quad P_{\psi} \mathrm{d} C_{2}=0 .
$$

Let us identify $M=\left(M_{1}, M_{2}, M_{3}\right)$ with the angular momentum vector of the Chaplygin sphere with respect to a contact point and the vector $\omega=\left(\omega_{1}, \omega_{2}, \omega_{3}\right)$

$$
\omega=A_{\gamma} M, \quad A_{\gamma}=A+\frac{d A \gamma \otimes \gamma A}{g^{2}}, \quad a_{k}=\left(I_{k}+d\right)^{-1}
$$

with the angular velocity vector of the rolling sphere. Its mass, inertia tensor and radius will be denoted by $m, I=\operatorname{diag}\left(I_{1}, I_{2}, I_{3}\right)$ and $b$, respectively. Parameter $d$ involves the mass and radius of the Chaplygin sphere $d=m b^{2}$, see [33, 11] for details.

In this case the bivector $P_{\psi}$, together with unvaried total mechanical energy $H$ (2.6)

$$
H=\frac{1}{2}(M, \omega)+V(\gamma)
$$

where $\omega$ is given by (2.17), generates the conformally Hamiltonian vector field

$$
X_{\psi}=g^{-1} P_{\psi} \mathrm{d} H, \quad H=\frac{1}{2}(M, \omega)+V(\gamma),
$$

endowed with the invariant measure

$$
\mu=g \mathrm{~d} \gamma \mathrm{d} M
$$

and having three first integrals $C_{1,2}$ and $H$. The corresponding equations of motion

$$
\dot{\gamma}=\gamma \times \omega, \quad \dot{M}=M \times \omega-\frac{\partial V}{\partial \gamma} \times \gamma
$$

describe the rolling of the Chaplygin sphere on a plane in a potential field [33, 11.

By adding an external nonconservative force we have to replace these equations with the equations

$$
\dot{\gamma}=\gamma \times \omega, \quad \dot{M}=M \times \omega+b \times \omega-\frac{\partial V}{\partial \gamma} \times \gamma,
$$

where the angular velocity vector is given by (2.17). 
Theorem 1 Equations (2.19) have an invariant measure

$$
\mu=g^{-1} \mathrm{~d} \gamma \mathrm{d} M
$$

It means that imposing the solenoidal field on the nonholonomic Chaplygin sphere preserves both the total mechanical energy (2.6) and the invariant measure (2.18).

The proof is a trivial observation that the equation on the last Jacoby multiplier

$$
\operatorname{div} \rho\left(\gamma_{1}, \gamma_{2}, \gamma_{3}\right) X_{\psi \varphi}=0
$$

is independent of the functions $b_{k}$ from the definition of the bivector $P_{\varphi}$. So, the solution of this equation can be used in a standard construction of the invariant measure simultaneously for the vector fields $X_{\psi}$ and $X_{\psi \varphi}$.

Composition $\psi \circ \varphi$ of the mappings $\varphi$ (2.7) and $\psi$ (2.15) reduces the original Lie-Poisson bivector $P(2.4)$ to the following Poisson bivector:

$$
P_{\psi \varphi}=g P_{\varphi}-\frac{d}{g}(M, A \gamma)\left(\begin{array}{cc}
0 & 0 \\
0 & \Gamma
\end{array}\right) .
$$

In fact, in order to get this expression, we have to replace $P$ with $P_{\varphi}$ in $P_{\psi}$ (2.16) and do not change $M_{i}$ in the second term. As above, this bivector satisfies the Jacobi condition only if condition (2.10) holds and has the same Casimir function as the bivector $P_{\varphi}$ (2.11)

$$
C_{1}=(\gamma, \gamma), \quad C_{2}=(\gamma, M+c), \quad P_{\psi \varphi} \mathrm{d} C_{1}=0, \quad P_{\psi \varphi} \mathrm{d} C_{2}=0 .
$$

Theorem 2 The equations of motion (2.19) in which the angular velocity vector $\omega$ is given by (2.17) are conformally Hamiltonian equations associated with the following vector field:

$$
X_{\psi \varphi}=g^{-1} P_{\psi \varphi} \mathrm{d} H, \quad H=\frac{1}{2}(M, \omega)+V(\gamma),
$$

generated by the total mechanics energy $H$ and by deformation of the Lie-Poisson brackets (2.3).

The proof consists of a direct verification.

Summing up, we prove the efficiency and relevance of the Dirac method in non-Hamiltonian mechanics using the nonholonomic Chaplygin sphere as an example. In a similar manner we can use compositions of the various known deformations of the Poisson brackets to construct equations of motion for other nonholonomic systems [5, 6, 9, 11, 33, 34, 36.

\section{Chaplygin sphere and Barnett-London effect}

It is well known that a magnetic body can be magnetized when rotating about one of its axes 28 . This magnetization by rotation was predicted and confirmed for ferromagnetic bodies by Barnett in 1915 [1. The same phenomenon was predicted in 1933 by Becker, Heller and Sauter 2] for a perfect conductor set into rotation. Subsequently London predicted that the same final state should result when a rotating normal metal is cooled into the superconducting state 29]. The resulting magnetic moment will depend on the shape of the body and is called the "London moment". A modern discussion of the dynamical understanding of the Meissner effect and the London moment may be found in 23., where the reader can also find references on experimental verifications of this effect for a variety of superconductors.

The 1980s witnessed many publications devoted to the problem of rigid body motion taking the Barnett - London effect into account 26, 27, 32, 39, 40, 41, see also the recent review 24] and references therein.

In this section we consider the motion of the nonholonomic Chaplygin sphere acted upon by a magnetic field, taking the Barnett - London effect into account. The corresponding equations of motion are equal to

$$
\dot{M}=(M+B \gamma+\alpha) \times \omega+\left(C \omega-\frac{\partial V}{\partial \gamma}\right) \times \gamma, \quad \dot{\gamma}=\gamma \times \omega .
$$


Here the entries of the angular velocity vector $\omega=\left(\omega_{1}, \omega_{2}, \omega_{3}\right)$ are arbitrary functions of coordinates $\gamma$ and $M, B$ and $C$ are $3 \times 3$ symmetric matrices, $\alpha=\left(\alpha_{1}, \alpha_{2}, \alpha_{3}\right)$ is the constant vector of gyrostatic moment, and the potential $V(\gamma)$ is a function on coordinates $\gamma$.

If $C=0$ and

$$
\omega=A M, \quad A=\left(\begin{array}{ccc}
a_{1} & 0 & 0 \\
0 & a_{2} & 0 \\
0 & 0 & a_{3}
\end{array}\right),
$$

the equations of motion are Hamiltonian equations [8]. In the case of the Grioli problem these equations describe the rotation of a dielectric rigid body with gyrostatic moment $\alpha \neq 0$ about a fixed point in a permanent homogeneous magnetic field and in a potential field [21, 22. In this case the matrix $B$ determines charge distribution, see [3, 8, 10, for details. For similar equations appearing in other mechanical models, see the textbooks [30, 31.

$$
\text { If } \alpha=0, B=0 \text { and }
$$

$$
\omega=A M
$$

Eqs. (3.22) are non-Hamiltonian equations describing the rotation of a ferromagnetic rigid body about a fixed point in a permanent homogeneous magnetic field and in a potential field, taking into account magnetization by rotation, see [32, 27] and Appendix D in [10. In this case, the matrix $C$ diagonal in the principal system of coordinates of the rigid body determines the anisotropy of ferromagnetic. Below we will consider an arbitrary symmetric matrix $C$.

If $\alpha=0, B=C=0$ and the angular velocity vector is given by (2.17)

$$
\omega=A_{\gamma} M, \quad A_{\gamma}=A+\frac{d A \gamma \otimes \gamma A}{g^{2}}, \quad g=\sqrt{1-d(\gamma, A \gamma)},
$$

the equations of motion (3.22) are conformally Hamiltonian equations describing the motion of the nonholonomic Chaplygin sphere on a plane, which we have considered in the previous section.

It is quite natural to study the motion of the Chaplygin sphere in a magnetic field when this sphere is made of a dielectric $B \neq 0$ or ferromagnetic material $C \neq 0$. So, below we substitute $\omega=A_{\gamma} M$ into (3.22) and find out which properties of the original nonholonomic system are preserved by imposing a magnetic field. This substitution $\omega=A_{\gamma} M$ can also be interpreted as imposing a suitable nonholonomic constraint on the well-studied dielectric and ferromagnetic rigid body in a magnetic field.

\subsection{Invariant measure and quadratic first integrals}

The equations of motion (3.22) have two so-called geometric first integrals which are independent of the form of the angular velocity vector

$$
J_{1}=(\gamma, \gamma), \quad J_{2}=(\gamma, M+\alpha)+\frac{1}{2}(\gamma, B \gamma)
$$

We can identify these first integrals with the Casimir functions of some deformations of the Lie-Poisson bivector (2.8), but in the generic case the corresponding vector field

$$
\frac{d x_{i}}{d t}=X_{i}, \quad i=1 \ldots 6, \quad x=(\gamma, M),
$$

is not a Hamiltonian vector field.

Theorem 3 At $\omega=A M$ the vector field $X$ (3.22) has an invariant measure

$$
\mu=\mathrm{d} \gamma \mathrm{d} M
$$

only if the matrix $C$ is a diagonal matrix

$$
C=\left(\begin{array}{ccc}
c_{1} & 0 & 0 \\
0 & c_{2} & 0 \\
0 & 0 & c_{3}
\end{array}\right)
$$


At $\omega=A_{g} M$ the vector field $X$ (3.22) has an invariant measure

$$
\mu=g^{-1} \mathrm{~d} \gamma \mathrm{d} M, \quad g=\sqrt{1-d(\gamma, A \gamma)},
$$

only if the matrix $C$ is a diagonal matrix with entries satisfying the Clebsch type condition

$$
\frac{c_{2}-c_{3}}{a_{1}}+\frac{c_{3}-c_{1}}{a_{2}}+\frac{c_{1}-c_{2}}{a_{3}}=0
$$

So, at $\omega=A M$ the well-known physical condition of diagonalization of $C$ in the principal system of coordinates of the rigid body coincides with the condition of the existence of an invariant measure.

Now let us come back to the generic symmetric matrix $C$ and substitute the total mechanical energy

$$
H=\frac{1}{2}(\omega, M)+V(\gamma)
$$

into the equation $X(H)=0$. Solving the resulting equation with respect to the entries of matrices $B, C$ and the entries of vector $\alpha$, one gets the following theorem.

Theorem 4 The total mechanical energy is a first integral of Eqs. (3.22) at $\omega=A M$ and at $\omega=A_{g} M$ only if the matrix $B$ and the vector $\alpha$ are arbitrary

$$
B=\left(\begin{array}{ccc}
b_{11} & b_{12} & b_{13} \\
b_{12} & b_{22} & b_{23} \\
b_{1,3} & b_{23} & b_{33}
\end{array}\right), \quad \alpha=\left(\alpha_{1}, \alpha_{2}, \alpha_{3}\right)
$$

and the matrix $C$ is proportional to the unit matrix

$$
C=\lambda E, \quad E=\left(\begin{array}{lll}
1 & 0 & 0 \\
0 & 1 & 0 \\
0 & 0 & 1
\end{array}\right), \quad \lambda \in \mathbb{R} .
$$

So, if the total mechanical energy is a first integral of Eqs. (3.22), then these equations have an invariant measure according to Theorem 3 . It is quite obvious because the total energy is altered only if the work done by external forces is equal to zero.

Substituting these conditions into the second part of the equations of motion (3.22), we can remove the "non-Hamiltonian" term $C \omega \times \gamma$ from these equations:

$$
\begin{aligned}
\dot{M} & =(M+B \gamma+\alpha) \times \omega+\left(C \omega-\frac{\partial V}{\partial \gamma}\right) \times \gamma=(M+B \gamma+\alpha) \times \omega+\left(\lambda \omega-\frac{\partial V}{\partial \gamma}\right) \times \gamma \\
& =(M+(B-\lambda E) \gamma+\alpha) \times \omega-\frac{\partial V}{\partial \gamma} \times \gamma
\end{aligned}
$$

see [27. The corresponding vector field $X$ is a Hamiltonian vector field at $\omega=A M$ and a conformally Hamiltonian vector field in the nonholonomic case $\omega=A_{\gamma} M$, see the previous section.

Now let us come back to the generic symmetric matrix $C$ and substitute the square of the angular momentum

$$
M^{2}=(M, M)=M_{1}^{2}+M_{2}^{2}+M_{3}^{2}
$$

into the equation $X\left(M^{2}\right)=0$. Solving the resulting equation with respect to the entries of the matrices $B, C$ and the entries of the vector $\alpha$, one gets the following theorem.

Theorem 5 The square of the angular momentum is a first integral of Eqs. (3.22) only if $\alpha=0$ and $V\left(\gamma_{1}, \gamma_{2}, \gamma_{3}\right)=0$.

At $\omega=A M$ the matrix $C$ has to be a diagonal matrix (3.25), whereas the matrix $B$ is equal to

$$
B=\left(\begin{array}{ccc}
\frac{a_{2} c_{2}-a_{3} c_{3}}{a_{2}-a_{3}} & 0 & 0 \\
0 & \frac{a_{3} c_{3}-a_{1} c_{1}}{a_{3}-a_{1}} & 0 \\
0 & 0 & \frac{a_{1} c_{1}-a_{2} c_{2}}{a_{1}-a_{2}}
\end{array}\right) .
$$


At $\omega=A_{\gamma} M$ the matrix $C$ has to be a diagonal matrix with entries satisfying (3.26), whereas the matrix $B$ is proportional to the unit matrix, for instance,

$$
B=\frac{a_{2} c_{2}-a_{3} c_{3}}{a_{2}-a_{3}}\left(\begin{array}{ccc}
1 & 0 & 0 \\
0 & 1 & 0 \\
0 & 0 & 1
\end{array}\right),
$$

up to permutation of indices.

So, if the square of the angular momentum is a first integral of Eqs. (3.22), then these equations have an invariant measure according to Theorem 3 ,

\subsection{Old and new partial cases of integrability by quadratures}

Let us consider a dynamically symmetric rigid body at $a_{1}=a_{2}$. In the nonholonomic case, i.e., at $\omega=A_{\gamma} M$, the first integral linear in $M_{i}$ does not exist.

At $\omega=A M$ Eqs. (3.22) have a standard invariant measure

$$
\mu=\mathrm{d} \gamma \mathrm{d} M
$$

only if

$$
C=\left(\begin{array}{ccc}
c_{11} & c_{12} & 0 \\
c_{12} & c_{22} & 0 \\
0 & 0 & c_{33}
\end{array}\right)
$$

in contrast with Theorem 3 for the generic case.

At $V=f\left(\gamma_{3}\right)$ Eqs. (3.22) have also a first integral which is a function linear in $M_{3}$

$$
K=M_{3}-c_{13} \gamma_{1}-c_{23} \gamma_{2}+\left(c_{11}-b_{2}\right) \gamma_{3}
$$

if $\alpha=\left(0,0, \alpha_{3}\right)$, the matrix $C$ is an arbitrary symmetric matrix

$$
C=\left(\begin{array}{lll}
c_{11} & c_{12} & c_{13} \\
c_{12} & c_{22} & c_{23} \\
c_{13} & c_{23} & c_{33}
\end{array}\right),
$$

and the matrix $B$ has the following form:

$$
B=\left(\begin{array}{ccc}
b_{1}+c_{22} & 0 & 0 \\
0 & b_{1}+c_{22} & 0 \\
0 & 0 & b_{3}+c_{33}
\end{array}\right)-C .
$$

In contrast with the first integrals quadratic in momenta $M_{i}$ from Theorems 4 and 5 the existence of a linear integral $K$ is not related to the existence of an invariant measure. For instance, if $c_{13} \neq 0$, there is a first integral $K$ linear in momenta, but there exists no invariant measure.

Equations of motion (3.22) have two so-called geometric first integrals (3.24), which are independent of the form of the angular velocity vector. In order to get a system completely integrable by quadratures, we have to find an invariant measure and two independent first integrals according to the Euler - Jacobi theorem. We can easily do it by combining conditions for the existence of an invariant measure, mechanical energy $H$, square of angular momenta $M^{2}$ and a first integral $K$.

For instance, condition (3.25) for the invariant measure leads to the standard form of the first integral

$$
K=M_{3}+\left(c_{11}-b_{1}\right) \gamma_{3} .
$$

By adding the condition from Theorem 4 one gets the integrable case from [32] with integrals of motion $K$ and

$$
H=\frac{1}{2}\left(a_{1} M_{1}^{2}+a_{1} M_{2}^{2}+a_{3} M_{3}^{2}\right)+V\left(\gamma_{3}\right) .
$$


Since $C=\lambda E$, transformation (3.27) reduces this system to the well-known integrable Kirchhoff case in the Grioli problem at

$$
B=\left(\begin{array}{ccc}
b_{1} & 0 & 0 \\
0 & b_{1} & 0 \\
0 & 0 & a_{3}
\end{array}\right)
$$

with gyrostatic moment $\alpha=\mu(0,0,1)[8,25,27$.

By adding the condition from Theorem 5 one gets an integrable case with integrals of motion $K$ and

$$
M^{2}=M_{1}^{2}+M_{2}^{2}+M_{3}^{2}
$$

at

$$
C=\left(\begin{array}{ccc}
a_{3} c_{3}+\frac{b_{1}\left(a_{1}-a_{3}\right)}{a_{1}} & 0 & 0 \\
0 & a_{3} c_{3}+\frac{b_{1}\left(a_{1}-a_{3}\right)}{a_{1}} & 0 \\
0 & 0 & a_{1} c_{3}
\end{array}\right), \quad \text { so that } \quad B=\left(\begin{array}{ccc}
b_{1} & 0 & 0 \\
0 & b_{1} & 0 \\
0 & 0 & a_{3}
\end{array}\right) \text {. }
$$

A particular case of this integrable system is discussed in Appendix D [10. We suppose that there is a change of variables which reduces this system to the same integrable Kirchhoff case of rigid body motion.

There are also two independent first integrals which are second-order polynomials in momenta $M_{i}$ if we have a completely symmetric rigid body

$$
a_{1}=a_{2}=a_{3} .
$$

In this particular case the vector field $X(3.22)$ always has an invariant measure

$$
\mu=\mathrm{d} \gamma \mathrm{d} M
$$

for $\omega=A M$ or $\omega=A_{\gamma} M$ without any restriction on the entries of the symmetric matrix $C$.

In this particular case at $\omega=A M=a M, \alpha=0$ and $V(\gamma)=0$ Eqs. (3.22)

$$
a^{-1} \dot{M}=B \gamma \times M+C M \times \gamma, \quad a^{-1} \dot{\gamma}=\gamma \times M
$$

involve two arbitrary symmetric matrices $B$ and $C$. Let us diagonalize the matrix $C$ using rotations of the vectors $M$ and $\gamma$

$$
C=\operatorname{diag}\left(c_{1}, c_{2}, c_{3}\right) .
$$

It is easy to see that Eqs. (3.22) with the diagonal matrix $C$ have the following first integral:

$$
F_{1}=\lambda_{1} M_{1}^{2}+\lambda_{2} M_{2}^{2}+\lambda_{3} M_{3}^{2}=(M, \Lambda M), \quad \Lambda=\left(\begin{array}{ccc}
\lambda_{1} & 0 & 0 \\
0 & \lambda_{2} & 0 \\
0 & 0 & \lambda_{3}
\end{array}\right)
$$

if

$$
B=\left(\begin{array}{ccc}
\frac{\lambda_{2} c_{3}-\lambda_{3} c_{2}}{\lambda_{2}-\lambda_{3}} & 0 & 0 \\
0 & \frac{\lambda_{1} c_{3}-\lambda_{3} c_{1}}{\lambda_{1}-\lambda_{3}} & 0 \\
0 & 0 & \frac{\lambda_{1} c_{2}-\lambda_{2} c_{1}}{\lambda_{1}-\lambda_{2}}
\end{array}\right) .
$$

Here $\lambda_{k} \in \mathbb{R}$ are arbitrary parameters.

If we impose additional restrictions on the matrices $B, C$ and $\Lambda$, we obtain two integrable cases of rigid body motion in a magnetic field. In the first case the additional restriction has the following form:

$$
\left(\lambda_{2}-\lambda_{3}\right) c_{1}+\left(\lambda_{3}-\lambda_{1}\right) c_{2}+\left(\lambda_{1}-\lambda_{2}\right) c_{3}=0 .
$$

If we solve this condition with respect to $c_{1}$

$$
c_{1}=\frac{\left(\lambda_{1}-\lambda_{3}\right) c_{2}-\left(\lambda_{1}-\lambda_{2}\right) c_{3}}{\lambda_{2}-\lambda_{3}}
$$


and substitute the resulting expression into the matrix $B(3.30)$, we see that the matrix $B$ is a unit matrix up to a scalar factor

$$
B=\frac{\lambda_{2} c_{3}-\lambda_{3} c_{2}}{\lambda_{2}-\lambda_{3}}\left(\begin{array}{lll}
1 & 0 & 0 \\
0 & 1 & 0 \\
0 & 0 & 1
\end{array}\right)
$$

It allows us to put $B=0$, i. e., $c_{k}=\lambda_{k}$, in Eqs. (3.29) without loss of generality. The corresponding first integral was found by V. V. Kozlov 27]

$$
F_{2}=(M, M)-2(M, C \gamma)+\left(\frac{\operatorname{tr}(C-B)}{\operatorname{tr} \Lambda}\right)^{2} \operatorname{det} \Lambda\left(\gamma, \Lambda^{-1} \gamma\right)
$$

In [38. Veselova proved that this system is equivalent to the well-known integrable Clebsch case for the Kirchhoff equations after some unobvious change of variables.

In the second case the additional restriction looks like

$$
\left(\lambda_{2}-\lambda_{3}\right) \lambda_{1} c_{1}+\left(\lambda_{3}-\lambda_{1}\right) \lambda_{2} c_{2}+\left(\lambda_{1}-\lambda_{2}\right) \lambda_{3} c_{3}=0
$$

If we solve this condition with respect to $c_{1}$

$$
c_{1}=\frac{\left(\lambda_{1}-\lambda_{3}\right) \lambda_{2} c_{2}-\left(\lambda_{1}-\lambda_{2}\right) \lambda_{3} c_{3}}{\lambda_{1}\left(\lambda_{2}-\lambda_{3}\right)}
$$

and substitute $c_{1}$ into the matrix $B(3.30)$, we obtain the following matrix:

$$
B=\frac{\lambda_{2} c_{3}-\lambda_{3} c_{2}}{\lambda_{2}-\lambda_{3}}\left(\begin{array}{ccc}
1 & 0 & 0 \\
0 & 1 & 0 \\
0 & 0 & 1
\end{array}\right)+\frac{c_{2}-c_{3}}{\left(\lambda_{2}-\lambda_{3}\right) \lambda_{1}}\left(\begin{array}{ccc}
0 & 0 & 0 \\
0 & \left(\lambda_{1}-\lambda_{2}\right) \lambda_{3} & 0 \\
0 & 0 & \left(\lambda_{1}-\lambda_{3}\right) \lambda_{2}
\end{array}\right),
$$

which cannot be removed from Eqs. (3.29). The additional first integral is equal to

$$
F_{2}=(M, M)-2(M, C \gamma)+\frac{\operatorname{tr}(C-B)}{\operatorname{tr} \Lambda^{-1}}\left(C \gamma, \Lambda^{-1} \gamma\right)
$$

We suppose that there is a counterpart of the Veselova transformation which reduces this system to the well-known integrable Steklov-Lyapunov case of rigid body motion. We plan to discuss this transformation in a forthcoming publication.

\section{Conclusion}

Thus, using the Chaplygin sphere as an example, we have proved the possibility and advisability of using Dirac's ideas not only in Hamiltonian classical and quantum mechanics, but also in nonholonomic mechanics. In addition, we have obtained a number of new results for the motion of the Chaplygin sphere on a plane in a constant magnetic field under the assumption that the sphere is made of a dielectric $B \neq 0$ and ferromagnetic $C \neq 0$ material. In a similar manner, using deformations of Poisson brackets and corresponding Poisson maps, one can also study various generalizations of the motion of the Chaplygin sphere [5, 36] and other nonholonomic systems [6, 9, 11, 34, 35] in a magnetic field.

As a development of the problems considered here, we also point out the possibility of introducing inhomogeneous and nonstationary magnetic fields [23, 26, 37. The first possibility is closely related to the Levitron theory and will allow the sphere, for example, to jump without additional mechanical actions. By contrast, the nonstationary field can in principle allow the sphere to be accelerated to the necessary velocity or the chaotic regime to be reached, which is diagnosed from intricate or even diffused motion of the contact point. All these processes should be studied from the viewpoint of mobile robots. An excellent review of these can be found in the dissertation [42].

A proof of unbounded growth of energy under periodic oscillations of the rotor inside the Chaplygin sleigh and of bounded growth in the presence of dissipation is given in [7]. An unbounded acceleration 
for the Chaplygin sphere is discussed in [13. We note that the acceleration mechanism of spheres is still not understood. The magnetic field, which is a complex gyroscopy, will allow progress in solving this problem.

In a number of instruments utilizing a contact less suspension system a rotor spins at high speed in a suspension magnetic field. The design of such instruments is feasible only if the properties of bodies rotating in a magnetic field are known [23, 26, 37. We could enrich this theory by taking into account various nonholonomic effects modelling friction.

The work of A. V. Tsiganov (Sections 1, 2) is supported by the Russian Science Foundation (project no. 19-71-30012) and performed in Steklov Mathematical Institute of Russian Academy of Sciences. The work of A. V. Borisov (Sections 3, 4) was carried out within the framework of the state assignment of the Ministry of Education and Science of Russia (1.2404.2017/4.6).

\section{References}

[1] Barnett, S. J., Magnetization by Rotation, Phys. Rev., 1915, vol.6, no. 4, pp. 239-270.

[2] Becker, R., Heller, G., and Sauter, F., Über die Stromverteilung in einer supraleitenden Kugel, Z. Phys., 1933, vol. 85, nos.11-12, pp. 772-787.

[3] Benvenuti, P. and Balli, R., Risolubilità per quadrature del problema del moto di un solido soggetto a forze di potenza nulla, Atti Accad. Nazion. Lincei, R. C. (8), Cl. Sci. Fis. Mat. Nat., 1974 , vol. 56, no. 1 , pp. $62-64$.

[4] Birtea, P., Caşu, I., and Comănescu, D., Hamilton - Poisson Formulation for the Rotational Motion of a Rigid Body in the Presence of an Axisymmetric Force Field and a Gyroscopic Torque, Phys. Lett. A, 2011, vol. 375, no. 45, pp. 3941-3945.

[5] Bizyaev, I. A., Borisov, A. V., and Mamaev, I. S., Different Models of Rolling for a Robot Ball on a Plane As a Generalization of the Chaplygin Ball Problem, Regul. Chaotic Dyn., 2019, vol. 24, no. 5 , pp. 560-582.

[6] Bizayev, I. A. and Tsiganov, A. V., On the Routh Sphere Problem, J. Phys. A, 2013, vol.46, 085202, $11 \mathrm{pp}$.

[7] Bizyaev, I. A., Borisov, A.V., Kozlov, V.V., and Mamaev, I. S., Fermi-Like Acceleration and Power-Law Energy Growth in Nonholonomic Systems, Nonlinearity, 2019, vol.32, no.9, pp. 3209-3233.

[8] Bogoyavlensky, O. I., Breaking Solitons. Nonlinear Integrable Equations, Moscow: Nauka, 1991 (Russian).

[9] Bolsinov, A. V., Borisov, A. V., and Mamaev, I. S., Geometrisation of Chaplygin's Reducing Multiplier Theorem, Nonlinearity, 2015, vol. 28, no. 7, pp. 2307-2318.

[10] Borisov, A. V. and Mamaev, I. S., Rigid Body Dynamics, De Gruyter Stud. Math. Phys., vol. 52, Berlin: De Gruyter, 2018.

[11] Borisov, A. V., Mamaev, I. S., and Tsyganov, A. V., Nonholonomic Dynamics and Poisson Geometry, Russian Math. Surveys, 2014, vol.69, no.3, pp.481-538; see also: Uspekhi Mat. Nauk, 2014, vol. 69 , no. 3, pp. 87-144.

[12] Borisov, A. V., Mamaev, I. S., and Bizyaev, I. A., Dynamical Systems with Non-Integrable Constraints: Vaconomic Mechanics, Sub-Riemannian Geometry, and Non-Holonomic Mechanics, Russian Math. Surveys, 2017, vol.72, no. 5, pp. 783-840; see also: Uspekhi Mat. Nauk, 2017, vol.72, no. 5(437), pp. 3-62.

[13] Borisov, A.V., Kilin, A.A., and Pivovarova, E. N., Speedup of the Chaplygin Top by Means of Rotors, Dokl. Phys., 2019, vol.64, no. 3, pp.120-124; see also: Dokl. Akad. Nauk, 2019, vol. 485, no. 3, pp. 285-289. 
[14] Dirac, P. A. M., Homogeneous Variables in Classical Dynamics, Math. Proc. Camb. Philos. Soc., 1933, vol. 29, no. 3, pp. 389-400.

[15] Dirac, P. A. M., Generalized Hamiltonian Dynamics, Proc. Roy. Soc. London Ser. A, 1958, vol. 246, no. 1246 , pp. $326-332$.

[16] Dirac, P. A. M., Lectures on Quantum Mechanics, Belfer Graduate School of Science Monographs Series, vol. 2, New York: Acad. Press, 1967.

[17] Dunne, G. V., Jackiw, R., Pi, S.-Y., and Trugenberger, C. A., Self-Dual Chern-Simons Solitons and Two-Dimensional Nonlinear Equations, Phys. Rev. D (3), 1991, vol. 43, no. 4, pp. 1332-1345.

[18] Fedorov, Yu. N. and García-Naranjo, L.C., The Hydrodynamic Chaplygin Sleigh, J. Phys. A, 2010, vol. 43, no. 43, 434013, 18 pp.

[19] Fedorov, Yu. N., García-Naranjo, L. C., and Vankerschaver, J., The Motion of the 2D Hydrodynamic Chaplygin Sleigh in the Presence of Circulation, Discrete Contin. Dyn. Syst., 2013, vol. 33, no. 9 , pp. 4017-4040.

[20] Flato, M., Lichnerowicz, A., and Sternheimer, D., Deformations of Poisson Brackets, Dirac Brackets and Applications, J. Mathematical Phys., 1976, vol.17, no. 9, pp. 1754-1762.

[21] Grioli, G., Moto attorno al baricentro di un giroscopio soggetto a forze di potenza nulla, Univ. Roma Ist. Naz. Alta Mat. Rend. Mat. e Appl. (5), 1947, vol. 6, pp. 439-463.

[22] Grioli, G., Sul moto di un corpo rigido asimmetrico soggetto a forze di potenza nulla, Rend. Sem. Mat. Univ. Padova, 1957, vol.27, pp. 90-102.

[23] Hirsch, J. E., Moment of Inertia of Superconductors, Phys. Lett. A, 2019, vol. 383, no. 1, pp. 83-90.

[24] Hussein, A. M., On the Motion of a Magnetized Rigid Body, Acta Mech., 2017, vol.228, no. 11, pp. 4017-4023.

[25] Kirchhoff, G. R., Über die Bewegung eines Rotationskörpers in einer Flüssigkeit, J. Reine Angew. Math., 1870, vol. 1870, no. 71, pp. 237-262.

[26] Kobrin, A. I. and Martynenko, Yu. G., Motion of a Conducting Solid Body near the Center of Mass in a Slowly Varying Magnetic Field, Sov. Phys. Dokl., 1981, vol. 26, no. 12, pp. 1134-1136; see also: Dokl. Akad. Nauk SSSR, 1981, vol. 261, no. 5, pp. 1070-1073.

[27] Kozlov, V.V., Problem of the Rotation of a Solid Body in a Magnetic Field, Izv. Akad. Nauk. Mekh. Tverd. Tela, 1985, vol. 20, no. 6, pp. 28-33 (Russian).

[28] Landau, L. D. and Lifshitz, E. M., Course of Theoretical Physics: Vol. 8. Electrodynamics of Continuous Media, Oxford: Pergamon, 1960.

[29] London, F., Superfluids: Vol. 1. Macroscopic Theory of Superconductivity, New York: Wiley, 1950.

[30] Marsden, J.E. and Ratiu, T.S., Introduction to Mechanics and Symmetry: A Basic Exposition of Classical Mechanical Systems, 2nd ed., Texts Appl. Math., vol. 17, New York: Springer, 1999.

[31] Marsden, J.E., Misiolek, G., Ortega, J.-P., Perlmutter, M., and Ratiu, T. S., Hamiltonian Reduction by Stages, Lecture Notes in Math., vol. 1913, Berlin: Springer, 2007.

[32] Samsonov, V. A., On the Rotation of a Body in a Magnetic Field, Izv. Akad. Nauk. Mekh. Tverd. Tela, 1984, vol.19, no. 4, pp. 32-34 (Russian).

[33] Tsiganov, A. V., Integrable Euler Top and Nonholonomic Chaplygin Ball, J. Geom. Mech., 2011, vol. 3, no. 3, pp. 337-362.

[34] Tsiganov, A.V., One Family of Conformally Hamiltonian Systems, Theoret. and Math. Phys., 2012, vol.173, no. 2, pp. 1481-1497; see also: Teoret. Mat. Fiz., 2012, vol.173, no. 2, pp.179-196. 
[35] Tsiganov, A. V., Poisson Structures for Two Nonholonomic Systems with Partially Reduced Symmetries, J. Geom. Mech., 2014, vol.6, no.3, pp. 417-440.

[36] Tsiganov, A. V., Hamiltonization and Separation of Variables for a Chaplygin Ball on a Rotating Plane, Regul. Chaotic Dyn., 2019, vol.24, no. 2, pp. 171-186.

[37] Urman, Yu. M., Influence of the Barnett-London Effect on the Motion of a Superconducting Rotor in a Nonuniform Magnetic Field, Tech. Phys., 1998, vol.43, no. 8, pp. 885-889; see also: Zh. Tekh. Fiz., 1998, vol.68, no. 8, pp.10-14.

[38] Veselova, L. E., About Two Problems in the Dynamics of a Rigid Body, Vestn. Mosk. Univ. Ser. 1. Mat. Mekh., 1986, no. 5, pp. 90-91 (Russian).

[39] Vitale, S., Bonaldi, M., Falferi, P., Prodi, G. A., and Cerdonio, M., Magnetization by Rotation and Gyromagnetic Gyroscopes, Phys. Rev. B, 1989, vol. 39, no. 16, pp. 11993-12002.

[40] Yegarmin, I. E., On the Magnetic Field of a Rotating Superconducting Body, in Aerophysics and Geocosmic Research, Moscow: MOTI, 1983, pp. 95-96 (Russian).

[41] Yehia, H.M., New Solutions of the Problem of Motion of Gyrostat in Potential and Magnetic Fields, Mosc. Univ. Mech. Bull., 1985, vol.50, no.5, pp.21-25; see also: Vestn. Mosk. Univ. Ser. 1. Mat. Mekh., 1985, no. 5, pp.60-63.

[42] Ylikorpi, T., Mobility and Motion Modelling of Pendulum-Driven Ball-Robots: Decoupled Models for Steering and Obstacle Crossing, Aalto University publication series Doctoral Dissertations 177/2017, available from https://aaltodoc.aalto.fi/handle/123456789/28401 (2017). 